\title{
The Dubious Authority of Maxwell's Equations in Free Space
}

\author{
C.R. MacCluer, Life Member, IEEE
}

\begin{abstract}
Because of the differential homogeneity of Maxwell's equations in free space, each solution can be additively perturbed in infinitely many ways into a physically unreasonable solution. Thus to be a solution holds no cachet. In particular, the traditional first example - the uniform plane wave - must be considered as only a metaphor.
\end{abstract}

Index Terms-Maxwell, plane wave, free space

\section{A RITE OF PASSAGE}

Generations of science and engineering students have labored through a course in electromagnetism, a course in three parts - electrostatics, where electric charges are held fixed; magnetics, where charges move at constant speed; and electromagnetics, where charges are accelerated.

Electrostatics has always been the most charming to me, with its list of standard geometric problems of charge and conductor placements. The resulting electrostatic fields and their potentials are elegantly deduced through clever use of the divergence theorem.

In part two, magnetic fields are mapped by clever use of Stokes' theorem. These techniques and computations of the first two parts, will appear and reappear in subsequent (at first glance) unrelated courses in mechanics, heat transfer, partial differential equations, thermodynamics, chemistry, and so on. These classical ideas have been the inspiration for much mathematics.

The triumphant third part of this traditional trilogy is electromagnetics, where by accelerating charges an experimenter can produce propagating wave fronts carrying energy that can do distant work. This electromagnetic phenomenon is believed to be completely described by the celebrated laws of Clerk Maxwell, first given as 20 equations in integral form, then soon after recast and condensed into four equations in differential form by Oliver Heaviside [8]. The publication of these four laws actually preceeded Hertz's demonstration of the physical existence of these propagating waves [8]. The four laws now hold a near mystical niche in the scientific pantheon, with extravagant praise such as "A glimpse into the mind of God" [6], or " Maxwell is the physicist's physicist" (Hawking), or "One scientific epoch ended and another began with James Clerk Maxwell" (Einstein). See [5]. Mawell's equations are thought to be an exact model, unlike (say) the heat equation, which although accurately models everyday heat transfer phenomenon, also predicts the unreasonable instantaneous transmission of information at an infinite distance [4], ex.1.35.

Emeritus Professor, Department of Mathematics, Michigan State University, East Lansing, MI, 48824 USA e-mail: maccluer@msu.edu.
One commonally heard extravagant claim is central to this article: "Whatever Maxwell allows, occurs," meaning that any mathematical solution, no matter how exotic - once the proper apparatus is constructed and experiment performed - will actually physically appear. But as we will now see, once an electromagnetic signal is emitted, it then propagates in (empty) free space where the four laws simplify and mathematical pathologies appear.

\section{MAXWELl'S EQUATIONS IN FREE SPACE}

Because there are no free charges or current flow, Maxwell's equations reduce to four homogeneous linear equations: The pair $(E, H)$ of vector fields, where $E$ is the electric field and $H$ is the magnetic field, satisfy

$$
\begin{gathered}
\nabla \cdot E=0, \\
\nabla \times H=\epsilon_{0} \frac{\partial E}{\partial t}, \\
\nabla \times E=-\mu_{0} \frac{\partial H}{\partial t}, \\
\nabla \cdot H=0 .
\end{gathered}
$$

COROLlary. The vector fields $E$ and $H$ satisfy

$$
\mu_{0} \epsilon_{0} \frac{\partial^{2} E}{\partial^{2} t}=\nabla^{2} E \quad \text { and } \quad \mu_{0} \epsilon_{0} \frac{\partial^{2} H}{\partial^{2} t}=\nabla^{2} H .
$$

The fundamental constants $\mu_{0}, \epsilon_{0}$ arose in earlier independent experiments of electrostatics, magnetics, and optics. Thus (2) is the stunning prediction that the speed of propagation of all electromagnetic waves, from radio to light, travels at the identical speed of $c=1 / \sqrt{\mu_{0} \epsilon_{0}}$.

Traditionally, the first lecture on electromagnetic waves in free space is the derivation of uniform plane waves.

\section{What IS A UNIFORM PLANE WAVE?}

A plane wave is a propagation mode, namely a solution pair $(E, H)$ to Maxwell's four equations (1a)-(1d), for which there exists some non-zero constant vector $P$ that is orthogonal to both $E$ and $H$ at each point $(x, y, z)$ and moment of time $t$.This plane wave is uniform if for each $t, E$ and $H$ are both constant throughout the propagating plane attached at $(x, y, z)$ perpendicular to $P$. 


\section{THE UNFORTUNATE FIRST FREE-SPACE LECTURE}

Invariably, plane waves in free space are derived by displaying Maxwell's equations simplified to their free space versions (1a)-(1d), then suddenly declaring that the electric field is some version of the complex exponential

$$
E=E_{0} \exp [i(\mathbf{k} \cdot \mathbf{r}-\omega t)]
$$

[1] sec. 9.2; [2], sec. 7.1; [7] . It is then a quick few steps to deducing that such plane wave fields are uniform, sinusoidal, and that the electric and magnetic fields are perpendicular and form a right-handed trihedral with the direction of travel none of which is true for the general plane wave solution. There is instead a rich zoo of plane wave solutions that are passed over by this traditional derivation.

\section{THE CANONICAL UNIFORM PLANE WAVE}

The first concrete example given of a plane wave is invariably the vector field pair

$$
E(x, y, z, t)=E(z, t)=\sqrt{\mu_{0}} \sin (\omega t-k z) \mathbf{i}
$$

and

$$
H(x, y, z, t)=H(z, t)=\sqrt{\epsilon_{0}} \sin (\omega t-k z) \mathbf{j},
$$

where the wave number $k=\omega / c$

Iindeed this pair $(E, H)$, propagating at speed $c=1 / \sqrt{\mu_{0} \epsilon_{0}}$ up the positive $z$-axis, staisfies the four free-space Maxwell equations (1a)-(1d). This (linearly polarized) solution is argueably the most common intuitive notion carried in the mind of every communications engineer. It, or a superposition with a quadrature version of itself, is the mode model of all transmitted radio, television, and optical communications [3]. Note that indeed $E$ and $H$ are orthogonal and form a righthanded trihedral with the direction of travel $\mathbf{k}$.

Embarrissingly, $(E, H+\mathbf{i})$ is also a solution pair that is no longer perpendicular. This trick has wider scope: Take any physically reasonable electromagnetic wave $(E, H)$ that has now escaped into free space. At time $t_{0}$ suppose $E$ points in the direction of the unit vector $\mathbf{u}$. Then the solution pair $(E, H+\mathbf{u})$ is not perpendiculat at this instant $t_{0}$.

Moreover, plane waves need not be uniform since we may perturb any solution pair by any time-invariant vector with divergence and curl 0 , as for example by $y \mathbf{i}+x \mathbf{j}$.

More damaging yet, because of the small numerical values of $\mu_{0}$ and $\epsilon_{0}$ in the SI system of units, by perturbing both $E$ and $H$ of (4) by $\mathbf{i}$ we obtain a trihedral with the direction of travel $\mathbf{k}$ that is at times left handed. In summary,

Warning. Every solution pair $(E, H)$ to Maxwell's equations in free space can be perturbed in infinitely many ways into a physically unreasonable solution pair. Thus being a solution to free-space Maxwell's equations carries no cachet.

\section{THE FINAL HUMILIATION OF THE UNIFORM PLANE WAVE}

Suppose $\Omega$ is a finite volume of space enclosed by its surface $\partial \Omega$. Then

$$
V=\int_{\Omega} \frac{\mu_{0} H^{2}+\epsilon_{0} E^{2}}{2} d \Omega
$$

is in fact the energy (Joules) stored within the volume $\Omega$ by the electric and magnetic fields at time $t,[2]$, eqn. (6.106).

Note that because the integrand of (7) - the energy density (Joules $/ \mathrm{m}^{3}$ ) — is nonnegative, the energy enclosed between any two propagating planes of a uniform plane wave is either zero or infinite. Thus the plane wave model does not reflect the finite energy that is found between expanding spherical wavefronts of actual electromagnetic emissions.

\section{SUMMING UP}

We must be more honest with our students. Because any solution pair $(E, H)$ to Maxwell's equations in free space can be additively perturbed in infinitely many ways to a solution pair with dubuous physical reality, the four equations alone are without authority. Some additional free-space conditions must be added to insure that solutions will be physically extant. In particular, the most familiar of all solutions, the linearly polarized sinusoidal travelling plane wave, must be considered only a metaphor.

\section{REFERENCES}

[1] D. Griffiths, Introduction to Electrodynamics, 4th ed.: Cambridge Univ. Press, 2014.

[2] J. Jackson, Classical Electrodynamics, 3rd ed., Wiley \& sons, 1998.

[3] J. Kraus, Antennas, McGraw-Hill, 1950.

[4] C. MacCluer, Boundary Value Problems and Fourier Expansions: Dover Publications, 2004.

[5] Maxwell quotes, AZ Quotes https://www.azquotes.com/quotes/topics/maxwell.html

[6] Personal communication with a UCLA doctorate plasma physicist, 2021.

[7] C. Papachristou, "Plane-wave solutions of Maxwell's equations: An educational note, Hellenic Naval Academy, https://metaconferences.org/omp/index.php/MP/catalog/book/70, 2020.

[8] J. Rautio, "The long road to Maxwell's equations," IEEE Spectrum, vol. 51, issue 12, 2014. https://spectrum.ieee.org/tech-history/dawn-ofelectronics/the-long-road-to-maxwells-equations 